\title{
A SECOND-ORDER INVARIANT OF THE NOETHER-LEFSCHETZ LOCUS AND TWO APPLICATIONS*
}

\author{
CATRIONA MACLEAN ${ }^{\dagger}$
}

Key words. Algebraic geometry, Noether-Lefyschetz problem

AMS subject classifications. $14 \mathrm{~N} 15$

1. Introduction and statement of results. Let $X \subset \mathbb{P}^{3}$ be a smooth surface of degree $d$ cut out by a polynomial

$$
F \in k\left[X_{0}, \ldots X_{3}\right]
$$

We will be interested in the following questions. What curves does $X$ contain? Can these curves be classified?

For a generic $X$ of degree $d \geq 4$, this question was answered in the 20's, when the Noether-Lefschetz theorem was proved by Lefschetz.

THEOREM 1 (Lefschetz). If $X$ is a generic smooth surface of degree $d \geq 4$ in $\mathbb{P}^{3}$ then for any curve $C \subset X$ there exists a surface $Y$ such that $C=X \cap Y$.

A curve $C$ which has the property that $C=X \cap Y$ for some surface $Y$ will be said to be a complete intersection in $X$.

This theorem says essentially that if $X$ is generic then the set of curves contained in $X$ is well understood and is as simple as possible. In this article we will study the distribution of surfaces for which the conclusion of Theorem 1 does not hold - or in other words, surfaces containing curves which are not well understood.

Throughout the rest of this article, we will denote by $U_{d}$ the space parameterising smooth degree $d$ surfaces in $\mathbb{P}^{3}$. We define the Noether-Lefschetz locus, which we denote by $N L_{d}$, as follows:

$X \in N L_{d} \Leftrightarrow X$ contains a curve $C$ which is not a complete intersection in $X$

which, by the Leftschetz $(1,1)$ theorem, can alternatively be written as

$$
X \in N L_{d} \Leftrightarrow H_{\text {prim }}^{1,1}(X, \mathbb{Z}) \neq 0
$$

Theorem 1 says that $N L_{d}$ is a countable union of proper subvarieties of $U_{d}$. Throughout the rest of the article, $N L$ will denote one of these subvarieties. Ciliberto et al. showed in [3] that $N L_{d}$ is dense in the Zariski and complex topologies.

It is interesting to have an idea of the size of the components of $N L_{d}$, since this gives us some idea of how rare badly-behaved curves are. An initial (very rough) estimate comes out of Hodge theory. Any component $N L$ can be expressed as the

\footnotetext{
* Received October 1, 2004; accepted for publication June 15, 2005.

${ }^{\dagger}$ Institut Fourier, 100 Rue des Mathématiques, Domaine Universitaire, Saint Martin d'Héres, Isére, France (Catriona.MacLean@ujf-grenoble.fr).
} 
zero locus of a section of a vector bundle of dimension $\left(\begin{array}{c}d-1 \\ 3\end{array}\right)$. Hence the codimension of $N L$ is at most $\left(\begin{array}{c}d-1 \\ 3\end{array}\right)$, and we expect that it will in fact be equal to $\left(\begin{array}{c}d-1 \\ 3\end{array}\right)$. If a component $N L$ has codimension strictly less than $\left(\begin{array}{c}d-1 \\ 3\end{array}\right)$, then we will say it is exceptional.

Since the dimension of $U_{d}$ is $\left(\begin{array}{c}d+3 \\ 3\end{array}\right)-1$, we expect $N L$ to be very small compared with $U_{d}$. Unfortunately, this bound is highly unsatisfactory, because in the simplest examples it fails to be exact by a very large margin. For example, the set of all surfaces containing a line is a Noether-Lefschetz locus of co-dimension $d-3$.

The principle that has guided much of the work on $N L_{d}$ is that very large components should be geometrically predictable. More precisely, the codimension estimate of $\left(\begin{array}{c}d-1 \\ 3\end{array}\right)$ was based on cohomological arguments, which do not take into account geometric information. Suppose $X$ contains a curve $C$ of low degree which is not a complete intersection in $X$. The Noether-Lefschetz locus corresponding to $C$ (which will be precisely defined in section 2.1), then has codimension $\leq H^{0}\left(\mathcal{O}_{C}(d)\right.$ ). This is much less than $\left(\begin{array}{c}d-1 \\ 3\end{array}\right)$ when $d \gg \operatorname{deg}(C)$.

The hope is that when a component $N L$ is large, this should always be explained by the presence of low-degree curves in the corresponding surfaces. Harris conjectured that the number of exceptional loci should be finite: Green and Ciliberto went further, proposing the following conjecture (which implies Harris's) and which we will call henceforth the Green-Ciliberto conjecture.

Conjecture 1 (Green-Ciliberto). If $\operatorname{codim}(N L)<\left(\begin{array}{c}d-1 \\ 3\end{array}\right)$, and $X$ is a point of $N L$ then there exists a curve $C \in X$ and a surface $Y \in \mathbb{P}^{3}$ of degree $\leq d-4$ such that

1. $C \subset X \cap Y$

2. $C$ is not a complete intersection in $X$.

We will discuss the motivation for this conjecture in section 5 . It has been proved by Voisin [16] that Harris's conjecture (and a fortiori the Green-Ciliberto conjecture) does not hold. However, it is interesting to ask whether a weakened version of the conjeture may hold. The main results which have been proved in this direction so far are the following.

- Voisin [13] and Green [6] prove that for $d \geq 5$ every exceptional NL component has codimension at least $d-3$, and for $d \geq 5$ this bound is obtained only for the component of surfaces containing a line.

- Voisin, [14] proves that for $d \geq 5$, the second largest NL component of $U_{d}$ has codimension $2 d-7$, and this bound is achieved only by the space of surfaces containing a conic.

- Otwinowska, [11] and [12], defines an analogue of $N L_{d}$ for hypersurfaces $X$ of a variety $Y$ of dimension $2 n+1$. She then proves that for any $b$, and for $d \gg b$, if $X \in N L$ has codimension $\leq \frac{b d^{n}}{n !}$, then $X$ contains an $n$-cycle of degree $\leq b$.

All of this work relies on a fundamental paper of Carlson and Griffiths [1], in which they give an algebraic expression for the tangent space of $N L_{d}$.

Our aim in this paper is to extend the results of Carlson and Griffiths via a second-order infinitesimal study of $N L$. After summarising the results of Carlson and Griffiths in section 2, we calculate in section 3 an invariant which, to- 
gether with the work of Carlson and Griffiths, describes the infinitesimal geometry of $N L$ at $X$ up to second order. This is the second-order invariant mentioned in the title.

This new invariant gives rise to a new family of equations when $X$ is a singular point of $N L$ or $N L$ is exceptional. In section 4 we will use these equations to prove Theorem 2, which completes the classification of exceptional Noether-Lefschetz loci in $U_{5}$ by finding all non-reduced components. (The reduced exceptional loci were determined by Voisin in [14]). In section 5 we will use them to prove Theorem 3 , which shows that a weakened version of the Green-Ciliberto conjecture holds for reduced Noether-Lefschetz loci.

Theorem 2. Let $N L$ be a non-reduced Noether-Lefschetz locus in $U_{5}$. The reduction of $N L$ is the space of all surfaces $X$ with the property that there exists a hyperplane $H$ such that $H \cap X$ contains two lines.

In Proposition 1 of section 4, we show that it is indeed the case that if $X$ has this property then $X$ lies on certain non-reduced Noether-Lefschetz loci. More precisely, if $L_{1}$ and $L_{2}$ are the two lines in question, and $\gamma=\alpha\left[L_{1}\right]_{\text {prim }}+\beta\left[L_{2}\right]_{\text {prim }}$, where $\alpha$ and $\beta$ are distinct non-zero rational numbers, then $N L(\gamma)$ is non-reduced.

In fact we will prove a stronger result, which is given in detail on page 12 (Theorem 8).

THEOREM 3. Suppose that $e \leq \frac{d-1}{2}$. There exists an integer, $\phi_{e}(d)$ such that if $N L$ is reduced, $X \in N L$ and codim $(N L) \leq \phi_{e}(d)$, then there exists a curve $C \in X$ and a surface $Y \in \mathbb{P}^{3}$ of degree e such that

1. $C \subset X \cap Y$

2. $C$ is not a complete intersection in $X$. Further, $\phi_{\frac{d-1}{2}}(d)=O\left(d^{3}\right)$.

Again, the result actually proved is somewhat stronger (see page 25, Theorem 9), but rather complicated to state.

\section{Preliminaries.}

2.1. Notation. Throughout the rest of this article, $\gamma$ will be a non-zero element of $H_{\text {prim }}^{1,1}(X, \mathbb{Z})$, and $O$ will be some contractible neighbourhood of $X$ in $U_{d}$. When $C$ is a curve in $X$ we will denote by $[C]_{\text {prim }}$ the primitive part of the cohomology class of $C$. When $\gamma$ is of the form $\sum_{i} \lambda_{i}\left[C_{i}\right]$ and $D \subset X$ has the property that $C_{i} \subset D$ for all $i$, we will say that $\gamma$ is supported on $D$. Unless otherwise stated, we will work over $O$. We now define $N L(\gamma)$, the Noether-Lefschetz locus associated to $\gamma$.

Let $\mathcal{H}^{i}$ be the vector bundle whose fibre over the point $X$ is $H^{i}(X, \mathbb{C})$. This vector bundle is equipped with the flat Gauss-Manin connection $\nabla$ and has a holomorphic structure. The Hodge filtration on $H^{i}(X, \mathbb{C})$ gives rise to a descending filtration $F^{p}\left(\mathcal{H}^{i}\right) \subset \mathcal{H}^{i}$ by holomorphic sub-vector bundles. We write $F^{p} / F^{p+1}=\mathcal{H}^{p, q}$. We denote by $\bar{\gamma}$ the section of $\left.\mathcal{H}^{2}\right|_{O}$ induced by flat transport of $\gamma$. There is a projection $\pi: \mathcal{H}^{2} \rightarrow \mathcal{H}^{0,2}$ and we denote $\pi(\bar{\gamma})$ by $\bar{\gamma}^{0,2}$. We now define:

Definition 1. The space $N L(\gamma)$ is the zero locus in $O$ of the section $\bar{\gamma}^{0,2}$.

By the Noether-Lefschetz locus associated to a curve $C$, we mean $N L\left([C]_{\text {prim }}\right)$. Any Noether-Lefschetz locus is locally equal to $N L(\gamma)$ for some $\gamma$. The Zariski tangent space to $N L(\gamma)$ was described by Carlson and Griffiths in [1]. 
2.2. The work of Carlson and Griffiths. In this section, we summarise the results of [8] and [1]. A summary of this work may also be found in [17].

Griffiths showed in [7] that

$$
\nabla\left(F^{p} \mathcal{H}^{i}\right) \subset F^{p-1}\left(\mathcal{H}^{i}\right) \otimes \Omega_{U_{d}}
$$

Quotienting, it follows that $\nabla$ induces an $\mathcal{O}_{U_{d}}$-linear map

$$
\bar{\nabla}: \mathcal{H}^{p, q} \rightarrow \mathcal{H}^{p-1, q+1} \otimes \Omega_{U_{d}} .
$$

For any $n, S^{n}$ will denote the space of degree $n$ homogeneous polynomials in variables $X_{0}, X_{1}, X_{2}, X_{3}$. Choose $P \in S^{p d-4}$ and let $\Omega$ be the canonical section of the bundle $K_{\mathbb{P}^{3}}(4)$. The form $\frac{P \Omega}{F^{p}}$ is then a holomorphic 3 -form on $\mathbb{P}^{3}-X$ and has a class in $H^{3}\left(\mathbb{P}^{3}-X, \mathbb{C}\right)$. The group $H^{3}\left(\mathbb{P}^{3}-X, \mathbb{C}\right)$ maps via the residue mapping res $X$ to $H_{\text {prim }}^{2}(X, \mathbb{C})$ : there is therefore in particular a composed mapping

$$
\operatorname{res}_{X}: S^{p d-4} \rightarrow H_{\text {prim }}^{2}(X, \mathbb{C})
$$

given by

$$
\operatorname{res}_{X}(P)=\operatorname{res}_{X}\left(\left[\frac{P \Omega}{F^{p}}\right]\right)
$$

It is proved in [8] (see also [1] and [17]) that

$$
\operatorname{Im}\left(\operatorname{res}_{X}\right)=F^{3-p} H_{\text {prim }}^{2}(X, \mathbb{C}),
$$

and that

$$
\operatorname{res}_{X}(Q) \in F^{2-p} H^{2}(X, \mathbb{C}) \text { if and only if } Q \in\left\langle\frac{\partial F}{\partial X_{0}}, \ldots, \frac{\partial F}{\partial X_{3}}\right\rangle .
$$

We denote by $J_{F}$ (the Jacobian ideal of $F$ ) the homogeneous ideal $\left\langle\frac{\partial F}{\partial X_{0}}, \ldots, \frac{\partial F}{\partial X_{3}}\right\rangle$. We further denote by $R_{F}$ (the Jacobian ring of $F$ ) the graded ring $k\left[X_{0} \ldots X_{3}\right] / J_{F}$. The results above can be summarised as follows.

TheOREM 4 (Carlson, Griffiths). The map resx induces a natural isomorphism between $R_{F}^{p d-4}$ and $H_{\text {prim }}^{3-p, p-1}(X, \mathbb{C})$.

In [1], the infinitesimal variation of this Hodge structure with variations of the hypersurface $X$ was also calculated. We have a map

$$
\bar{\nabla}: \mathcal{H}_{\mathrm{prim}}^{p, q} \rightarrow \operatorname{Hom}\left(T_{U_{d}}, \mathcal{H}_{\mathrm{prim}}^{p-1, q+1}\right) .
$$

Carlson and Griffiths showed that after making the following identifications

1. $T_{U_{d}}(F)=S^{d} /\langle F\rangle$,

2. $\mathcal{H}_{\mathrm{prim}}^{p, q}(F)=R_{F}^{(3-p) d-4}$,

3. $\mathcal{H}_{\text {prim }}^{p-1, q+1}(F)=R_{F}^{(4-p) d-4}$, 
we have the following result.

TheOREM 5 (Carlson, Griffiths). Up to multiplication by a constant, $\bar{\nabla}_{F}\left(\operatorname{res}_{X} P\right)$ is identified with the multiplication map

$$
\cdot P: R_{F}^{d} \rightarrow R_{F}^{(4-p) d-4}
$$

Henceforth, $P$ will denote an element of $S^{2 p-4}$ such that $\operatorname{res}_{X}(P)=\gamma$. We have the following description of the tangent space to $N L(\gamma)=\operatorname{zero}\left(\bar{\gamma}^{0,2}\right)$.

$$
T_{N L(\gamma)}(X)=\operatorname{Ker}\left(\cdot P: R_{F}^{d} \rightarrow R_{F}^{3 d-4}\right) .
$$

or in other words

$$
H \in T_{N L(\gamma)}(X) \text { if and only if there exist } Q_{i} \in S^{2 d-3} \text { such that } P H=\sum_{i=0}^{3} Q_{i} \frac{\partial F}{\partial X_{i}} .
$$

We will lean heavily in what follows on the following classical result, due to Macaulay (which may be found in [4], for example).

Theorem 6 (Macaulay). The ring $R_{F}$ is a Gorenstein graded ring. In other words, $R_{F}^{4 d-8}=\mathbb{C}$ and the multiplication map

$$
R_{F}^{a} \otimes R_{F}^{4 d-8-a} \rightarrow R_{F}^{4 d-8}=\mathbb{C}
$$

is a perfect pairing.

3. The second order invariant of IVHS. Throughout the rest of this article, $G$ and $H$ will be degree $d$ polynomials contained in $T_{N L(\gamma)}(X)$, and $\left\{Q_{i}\right\}_{i=0}^{3},\left\{R_{i}\right\}_{i=0}^{3}$ will be degree $2 d-3$ polynomials such that

$$
P G=\sum_{i=0}^{3} Q_{i} \frac{\partial F}{\partial X_{i}} \text { and } P H=\sum_{i=0}^{3} R_{i} \frac{\partial F}{\partial X_{i}}
$$

We will extend the work of Carlson and Griffiths to second order using the fundamental quadratic form of a section of a vector bundle- a generalisation of the Hessian, which we now briefly recall.

Let $M$ be a smooth $m$-dimensional complex scheme, $V$ a rank- $r$ vector bundle on $M$ and $\sigma$ a section of $V$. We denote by $W$ the zero scheme of $\sigma$ and choose a point $x$ of $W$. We choose also holomorphic co-ordinates, $z_{1}, \ldots, z_{m}$, on some neighbourhood of $x$ and a trivialisation of $V$ near $x$. Having picked such trivialisations, $\sigma$ becomes an $r$-tuple of holomorphic functions $\left(\sigma_{1}, \sigma_{2} \ldots \sigma_{r}\right)$. We define the map

$$
d \sigma_{x}: T_{U}(x) \rightarrow V_{x}
$$

by

$$
d \sigma_{x}\left(\sum_{i=1}^{m} \alpha_{i} \frac{\partial}{\partial z_{i}}\right)=\sum_{i=1}^{n} \alpha_{i} \frac{\partial \sigma}{\partial z_{i}}
$$


It can be shown that this map is independent of the choice of trivialisation and of local co-ordinates. The space $\operatorname{Ker}\left(d \sigma_{x}\right)$ is the Zariski tangent space to $W$ at $x$. We define the fundamental quadratic form, $q_{\sigma, x}$, of $\sigma$ at $x$ as follows.

$$
q_{\sigma, x}: T_{W}(x) \otimes T_{W}(x) \rightarrow V_{x} / \operatorname{Im}\left(d \sigma_{x}\right)
$$

is defined by

$$
q_{\sigma, x}\left(\sum_{i=1}^{m} \alpha_{i} \frac{\partial}{\partial z_{i}}, \sum_{j=1}^{m} \beta_{j} \frac{\partial}{\partial z_{j}}\right)=\sum_{i=1}^{m} \alpha_{i} \frac{\partial}{\partial z_{i}}\left(\sum_{j=1}^{m} \beta_{j} \frac{\partial}{\partial z_{j}}(\sigma)\right) .
$$

This, similarly, is independent of the choice of local trivialisation of $V$ and the choice of local co-ordinates $z_{j}$.

REMARK 1. If $x$ is a smooth point of $W_{\mathrm{red}}$ and $\operatorname{rk}(\operatorname{Ker}(d \sigma))$ is constant in a neighbourhood of $x$, then $q(u, w)=0$ for any $u \in T_{W_{\mathrm{red}}}$. Indeed, we may choose local co-ordinates on $U$ in such a way that $w=\frac{\partial}{\partial z_{1}}$ and $\left.\frac{\partial \sigma}{\partial z_{1}}\right|_{W_{\mathrm{red}}}=0$.

As an example, if $M$ is the space $\mathbb{C}^{2}, V$ is the trivial vector bundle $\mathbb{C}$ and $\sigma$ is the section $x y$, then the space $V_{x} / \operatorname{Im}\left(d \sigma_{x}\right)$ is non-zero only at the point $x=(0,0)$ and the form $q_{\sigma, x}: \mathbb{C}^{2} \otimes \mathbb{C}^{2} \rightarrow \mathbb{C}$ is given by

$$
q((a, b),(c, d))=a c \frac{\partial^{2} x y}{\partial x \partial x}+a d \frac{\partial^{2} x y}{\partial x \partial y}+b c \frac{\partial^{2} x y}{\partial y \partial x}+b d \frac{\partial^{2} x y}{\partial y \partial y}=a d+b c .
$$

We are now in a position to state our result.

THEOREM 7. The fundamental quadratic form

$$
q_{\bar{\gamma}, X}: \operatorname{Sym}^{2}\left(T_{N L(\gamma)}(X)\right) \rightarrow R_{F}^{3 d-4} / \operatorname{Im}(\cdot P)
$$

is given by

$$
q(G, H)=\sum_{i=0}^{3}\left(H \frac{\partial Q_{i}}{\partial X_{i}}-R_{i} \frac{\partial G}{\partial X_{i}}\right)
$$

The attentive reader will be surprised to see that this form is apparently not symmetric in $G$ and $H$. This is, however, only apparent: we have the following lemma.

Lemma 1. For all $H$ and $G$ in $T_{N L(\gamma)}(X)$,

$$
q(G, H)=q(H, G) .
$$

Proof of Lemma 1. We know that

$$
\sum_{i=0}^{3} G R_{i} \frac{\partial F}{\partial X_{i}}=G H P=\sum_{i=0}^{3} H Q_{i} \frac{\partial F}{\partial X_{i}}
$$

Rearranging, we get that

$$
\sum_{i=0}^{3}\left(G R_{i}-H Q_{i}\right) \frac{\partial F}{\partial X_{i}}=0 .
$$

Since the $\frac{\partial F}{\partial X_{i}}$ form a regular sequence, there exist $A_{i, j}$, polynomials, such that 
1. $A_{i, j}=-A_{j, i}$,

2. $G R_{i}-H Q_{i}=\sum_{j=0}^{3} A_{i, j} \frac{\partial F}{\partial X_{i}}$.

Deriving this second equation and summing over $i$, we get that

$$
\sum_{i=0}^{3}\left(G \frac{\partial R_{i}}{\partial X_{i}}+R_{i} \frac{\partial G}{\partial X_{i}}\right)-\sum_{i=0}^{3}\left(H \frac{\partial Q_{i}}{\partial X_{i}}-Q_{i} \frac{\partial H}{\partial X_{i}}\right)=\sum_{i, j}\left(\frac{\partial A_{i, j}}{\partial X_{i}} \frac{\partial F}{\partial X_{i}}+A_{i, j} \frac{\partial F}{\partial X_{i} \partial X_{j}}\right) .
$$

From this we deduce that

$$
\sum_{i=0}^{3}\left(G \frac{\partial R_{i}}{\partial X_{i}}+R_{i} \frac{\partial G}{\partial X_{i}}\right)-\sum_{i=0}^{3}\left(H \frac{\partial Q_{i}}{\partial X_{i}}+Q_{i} \frac{\partial H}{\partial X_{i}}\right) \in\left\langle\frac{\partial F}{\partial X_{i}}\right\rangle .
$$

This completes the proof of Lemma 1.

3.1. The fundamental quadratic form: an explicit description (proof of theorem 7). Recall that $G, H$ are elements of $T_{N L(\gamma)}(X)$. When $f$ is a section of a vector bundle vanishing at $X$, we will denote by $\frac{\partial f}{\partial G}(X)$ the derivative of $f$ along the tangent vector $G$ at the point $X$. We have that:

$$
q_{\bar{\gamma}^{0,2}, X}(G, H)=\frac{\partial\left(d \bar{\gamma}^{0,2}(H)\right)}{\partial G}(X),
$$

where $d \bar{\gamma}^{0,2}$ is as defined in 1 This equation is an equality between elements of the space $H^{0,2}(X, \mathbb{C}) / \operatorname{Im}\left(d \bar{\gamma}^{0,2}\right)$.

We choose $s$ a section of $S^{2 d-4} \otimes \mathcal{O}_{N L(\gamma)}$ such that $\operatorname{res}_{\tilde{X}}(s(\tilde{X}))=\bar{\gamma}(\tilde{X})$. After identification of $\mathcal{H}_{\mathrm{prim}}^{3-p, p-1}$ and $R_{F}^{p d-4}$ we have that

1. $\operatorname{Im}\left(d \bar{\gamma}^{0,2}(X)\right)=\operatorname{Im}(\cdot P)$

2. $d \bar{\gamma}^{0,2}(H)(\tilde{X})=H s(\tilde{X})$.

and hence

$$
q_{\bar{\gamma}^{0,2}, X}(G, H)=\frac{\partial\left(\operatorname{res}_{\tilde{X}}(H s(\tilde{X}))\right)}{\partial G}(X),
$$

this last equation being an equality between elements of $R_{F}^{3 p-4} / \operatorname{Im}(\cdot P)$.

Let us explain more precisely what we mean by the formula (2). Since $H s(\tilde{X})$ is a degree $3 d-4$ polynomial, it has a residue class $\operatorname{res}_{\tilde{X}}(H s(\tilde{X}))$ in $H^{0,2}(\tilde{X})$. This class disappears at $X$, and $\frac{\partial\left(\operatorname{res}_{\tilde{X}}(H s(\tilde{X}))\right)}{\partial G}(X)$ denotes its derivation along the tangent vector $G \in T_{U_{d}}(X)$. We note that

$$
\frac{\partial\left(\operatorname{res}_{\tilde{X}}(H s(\tilde{X}))\right)}{\partial G}(X)=\operatorname{res}_{X}\left(H \frac{\partial s(\tilde{X})}{\partial G}(X)\right)+\frac{\partial\left(\operatorname{res}_{\tilde{X}}(H P)\right)}{\partial G}(X) .
$$

Lemma 2. We have $\frac{\partial\left(\operatorname{res}_{\tilde{X}}(H P)\right)}{\partial G}(X)=-\operatorname{res}_{X}\left(\sum_{i=0}^{3} R_{i} \frac{\partial G}{\partial X_{i}}\right)$.

Proof of Lemma 2. If $X_{\epsilon}$ is the variety cut out by the polynomial $F+\epsilon G$, then we have

$$
\frac{\partial\left(\operatorname{res}_{X_{\epsilon}}(H P)\right)}{\partial \epsilon}(0)=\lim _{\epsilon \rightarrow 0} \frac{1}{\epsilon} \operatorname{res}_{X_{\epsilon}}(H P) .
$$


We know that $H P=\sum_{i=0}^{3} R_{i} \frac{\partial F}{\partial X_{i}}$, whence we see that

$$
H P=\sum_{i=0}^{3}\left(R_{i} \frac{\partial F+\epsilon G}{\partial X_{i}}-\epsilon R_{i} \frac{\partial G}{\partial X_{i}}\right) .
$$

Therefore,

$$
\operatorname{res}_{X_{\epsilon}}(H P)=\operatorname{res}_{X_{\epsilon}}\left(-\epsilon \sum_{i=0}^{3} R_{i} \frac{\partial G}{\partial X_{i}}\right)
$$

and hence

$$
\frac{\partial\left(\operatorname{res}_{\tilde{X}}(H P)\right)}{\partial G}(X)=\frac{\partial\left(\operatorname{res}_{X_{\epsilon}}(H P)\right)}{\partial \epsilon}(X)=\lim _{\epsilon \rightarrow 0} \operatorname{res}_{X_{\epsilon}}\left(-\sum_{i=0}^{3} R_{i} \frac{\partial G}{\partial X_{i}}\right) .
$$

From this we get that

$$
\frac{\partial\left(\operatorname{res}_{\tilde{X}}(H P)\right)}{\partial G}(X)=\operatorname{res}_{X}\left(-\sum_{i=0}^{3} R_{i} \frac{\partial G}{\partial X_{i}}\right) .
$$

This completes the proof of Lemma 2.

It remains to calculate $\frac{\partial s}{\partial G}(X)$.

Lemma 3. The section s can be chosen in such a way that $\frac{\partial s}{\partial G}(X)=\sum_{i=0}^{3} \frac{\partial Q_{i}}{\partial X_{i}}$.

Proof of Lemma 3. By definition

$$
\operatorname{res}_{X}(P)=\operatorname{res}_{X}\left[\frac{P \Omega}{F^{2}}\right] .
$$

The polynomial $s(\tilde{X})$ is chosen such that the section $\operatorname{res}_{\tilde{X}}(s(\tilde{X}))=\operatorname{res}_{\tilde{X}} \frac{s(\tilde{X}) \Omega}{\tilde{F}^{2}}$ of $\mathcal{H}^{2} \otimes \mathcal{O}_{N L(\gamma)}$ is flat with respect to the Gauss-Manin connection. In particular,

$$
\frac{\partial\left(\operatorname{res}_{\tilde{X}}(s(\tilde{X}))\right)}{\partial G}(X)=0
$$

and hence

$$
\operatorname{res}_{X}\left(\frac{\partial \frac{s \Omega}{F^{2}}}{\partial G}(X)\right)=0
$$

On deriving this formula, we obtain that

$$
\operatorname{res}_{X}\left(\frac{\left(\frac{\partial s}{\partial G}(X)\right) \Omega}{F^{2}}-2 \frac{G P \Omega}{F^{3}}\right)=0 .
$$

It is proved in [2] that (3) only holds if there is an $\alpha \in H^{0}\left(\Omega_{\mathbb{P}^{3}}^{2}(2 Y)\right)$ such that

$$
\frac{\frac{\partial s}{\partial G}(X) \Omega}{F^{2}}-2 \frac{G P \Omega}{F^{3}}=d \alpha .
$$


Any $\alpha \in H^{0}\left(\Omega_{\mathbb{P}^{3}}^{2}(2 Y)\right)$ may be written in the form

$$
\alpha=\frac{\sum_{i=0}^{3} S_{i} \operatorname{int}\left(\frac{\partial}{\partial X_{i}}\right) \Omega}{F^{2}}
$$

where the $S_{i}$ are degree $2 d-3$ polynomials. Here, the operation int $T_{Y} \otimes \Omega_{Y}^{2} \rightarrow \Omega_{Y}^{1}$ is defined for any smooth variety $Y$ by $\operatorname{int}(t, \omega)(v)=(\omega(t, v))$. We now show that

$$
d \alpha=\frac{-2}{F^{3}} \sum_{i=0}^{3} S_{i} \frac{\partial F}{\partial X_{i}} \Omega+\frac{1}{F^{2}} \sum_{i=0}^{3} \frac{\partial S_{i}}{\partial X_{i}} \Omega .
$$

We shall do this by calculation on $\mathbb{C}^{4}$. There is a natural application $\pi: \mathbb{C}^{4} \rightarrow \mathbb{P}^{3}$ given by $\left(x_{0}, \ldots, x_{3}\right) \rightarrow\left[x_{0}, \ldots, x_{3}\right]$. The pullback $\pi^{*}(\Omega)$ is given by

$$
\pi^{*}(\Omega)=\operatorname{int}\left(\sum_{j=0}^{3} x_{j} \frac{\partial}{\partial x_{j}}, d x_{0} \wedge \ldots \wedge d x_{3}\right)
$$

and the pullback $\pi^{*} \alpha$ is given by

$$
\pi^{*}(\alpha)=\sum_{i=0}^{3} \frac{S_{i}}{F^{2}} \operatorname{int}\left(\frac{\partial}{\partial x_{i}}, \operatorname{int}\left(\sum_{j=0}^{3} x_{j} \frac{\partial}{\partial x_{j}}, d x_{0} \wedge \ldots \wedge d x_{3}\right)\right) .
$$

We now consider (for example) $U_{3}$, the open set of $\mathbb{P}^{3}$ given by $X_{3} \neq 0$, and we map it into $\mathbb{C}^{4}$ via the map

$$
s:\left[X_{0}, \ldots, X_{3}\right] \rightarrow\left(X_{0} / X_{3}, X_{1} / X_{3}, X_{2} / X_{3}, 1\right) .
$$

The coordinates $X_{0} / X_{3}, X_{1} / X_{3}, X_{2} / X_{3}$ on $U_{3}$ will be denoted by $x_{0}, \ldots, x_{2}$. The map $s$ is a section of $\pi$. We therefore have that $s^{*} \circ \pi^{*}(\alpha)=\left.\alpha\right|_{U_{3}}$. Therefore

$$
\left.\alpha\right|_{U_{3}}=\left(\sum_{i=0}^{2}-(1)^{i+1} \frac{S_{i}}{F^{2}}+(-1)^{i} X_{i} \frac{S_{3}}{F^{2}}\right)\left(x_{0}, \ldots, x_{2}, 1\right) d x_{0} \wedge \ldots \hat{x_{i}} \ldots \wedge d x_{2} .
$$

It follows that

$$
\left.d \alpha\right|_{U_{3}}=\sum_{i=0}^{2}\left(-\frac{\partial \frac{S_{i}}{F^{2}}}{\partial X_{i}}+\frac{\partial \frac{X_{i} S_{3}}{F^{2}}}{\partial X_{i}}\right)\left(x_{0}, \ldots, x_{2}, 1\right) d x_{0} \wedge \ldots \wedge d x_{2},
$$

and hence

$$
\left.d \alpha\right|_{U_{3}}=\left(\sum_{i=0}^{3}-\frac{\partial \frac{S_{i}}{F^{2}}}{\partial X_{i}}+\sum_{i=0}^{3} X_{i} \frac{\partial \frac{S_{3}}{F^{2}}}{\partial X_{i}}+\sum_{i=0}^{2} \frac{S_{3}}{F^{2}}\right)\left(x_{0}, \ldots, x_{2}, 1\right) d x_{0} \wedge \ldots \wedge d x_{2} .
$$

By the Euler relationship, plus the fact that the degree of $\frac{S_{3}}{F^{2}}$ is -3 , it follows that

$$
\begin{gathered}
\left.d \alpha\right|_{U_{3}}=\sum_{i=0}^{3}-\frac{\partial \frac{S_{i}}{F^{2}}}{\partial X_{i}}\left(x_{0}, \ldots, x_{2}, 1\right) d x_{0} \wedge \ldots \wedge d x_{2} \\
\left.d \alpha\right|_{U_{3}}=\sum_{i=0}^{3} \frac{\partial \frac{S_{i}}{F^{2}}}{\partial X_{i}} \Omega
\end{gathered}
$$


and hence, as required

$$
d \alpha=\frac{-2}{F^{3}} \sum_{i=0}^{3} S_{i} \frac{\partial F}{\partial X_{i}} \Omega+\frac{1}{F^{2}} \sum_{i=0}^{3} \frac{\partial S_{i}}{\partial X_{i}} \Omega .
$$

Recall that

$$
\sum_{i=0}^{3} Q_{i} \frac{\partial F}{\partial X_{i}}=G P
$$

Therefore, the equation

$$
\frac{\left(\left(\frac{\partial s}{\partial G}(X)\right) \Omega\right)}{F^{2}}-2 \frac{H P \Omega}{F^{3}}=d \alpha
$$

is satisfied whenever

$$
\frac{\partial s}{\partial G}(X)=\sum_{i=0}^{3} \frac{\partial Q_{i}}{\partial X_{i}}
$$

and

$$
\alpha=\frac{\sum_{i=0}^{3} Q_{i} \operatorname{int}\left(\frac{\partial}{\partial X_{i}}\right) \Omega}{F^{2}} .
$$

Since the kernel of the map $S^{6} \otimes \mathcal{O}_{U_{d}} \rightarrow \mathcal{H}^{2}$ is of constant rank, it follows that we may choose $s$ such that $\frac{\partial s}{\partial G}(X)=\sum_{i=0}^{3} \frac{\partial Q_{i}}{\partial X_{i}}$. This completes the proof of Lemma 3.

It follows that

$$
\frac{\partial d_{H}\left(\bar{\gamma}^{0,2}\right)}{\partial G}(X)=\operatorname{res}_{X}\left(\sum_{i=0}^{3}\left(\frac{\partial Q_{i}}{\partial X_{i}} H-R_{i} \frac{\partial G}{\partial X_{i}}\right)\right) .
$$

Therefore $q_{\bar{\gamma}^{0,2}, X}(H, G)$ is equal to

$$
\sum_{i=0}^{3}\left(\frac{\partial Q_{i}}{\partial X_{i}} H-R_{i} \frac{\partial G}{\partial X_{i}}\right)
$$

As always, this is of course an equality of elements of $R_{F}^{3 d-4} / \operatorname{Im}(\cdot \mathrm{P})$. This completes the proof of Theorem 7 .

4. Non-reduced Noether-Lefschetz loci in $U_{5}$ (proof of theorem 2). We will actually prove the following, which is slightly more precise.

Theorem 8. Let $N L(\gamma) \subset U_{5}$ be non-reduced. Let $X$ be a point of $N L(\gamma)$. Then there exist $H$ a hyperplane, $L_{1}, L_{2}$ distinct lines in $X \cap H$ and $\alpha, \beta$ distinct non-zero rational numbers such that

$$
\gamma=\alpha\left[L_{1}\right]_{\text {prim }}+\beta\left[L_{2}\right]_{\text {prim }} .
$$

Traditionally, non-reduced Noether-Lefschetz components have been hard to study, since the much-used technique of degenerating $X$ relies on being able to 
integrate vector fields. We will use a different approach. The equations arising from the fundamental quadratic form allow us to directly construct harmonic forms on the complement of a special hyperplane section of $X$. The existence of such harmonic forms implies this section is reducible.

When $d=5$, any component of the Noether-Lefschetz locus has codimension at most 4. It was proved in [13], [6] that the codimension of $N L(\gamma)$ is $\geq 2$ and this bound is achieved only if $\gamma$ is a multiple of $\left[L_{1}\right]_{\text {prim }}$ for some line $L_{1} \subset X$. Further, it was shown in [14] that if $N L(\gamma)$ is of codimension 3, then $\gamma$ is a multiple of $\left[C_{1}\right]_{\text {prim }}$ for some conic $C_{1} \subset X$.

The only other Noether-Lefschetz loci in $U_{5}$ which may have tangent spaces with exceptional codimension are non-reduced components, whose reductions are of codimension 4.

Proposition 1. Assume there exists a hyperplane $H$ whose intersection with $X$ has 3 components $L_{1}, L_{2}, C$ such that $L_{1}$ and $L_{2}$ are distinct lines and $C$ is a cubic. If $\alpha$ and $\beta$ are distinct non-zero integers, then the cohomology class

$$
\gamma=\alpha\left[L_{1}\right]_{\text {prim }}+\beta\left[L_{2}\right]_{\text {prim }}
$$

is such that $N L(\gamma)$ has a non-reduced component.

Proof of Proposition 1. Since $\alpha, \beta$ are distinct and non-zero, $\gamma$ is neither the (primitive part of a) class of a line nor the (primitive part of a) class of a conic. We know by the work of Voisin in [15] that $\operatorname{codim}\left(T_{N L_{\gamma}}(X)\right)>3$, and hence codim $\left.T_{N L(\gamma)_{\text {red }}}(X)\right)=4$. We now show that $N L(\gamma)$ has a non-reduced component.

The space $N L(\gamma)$ contains the space $N L\left(L_{1_{\text {prim }}}\right) \cap N L\left(L_{2_{\text {prim }}}\right)$. Since this set has codimension $\leq 2+2=4$, it follows that $N L\left(L_{1_{\text {prim }}}\right) \cap N L\left(L_{2_{\text {prim }}}\right)$ is a component of $N L(\gamma)$.

A dimension count shows that for all $Y \in N L\left(C_{1_{\text {prim }}}\right)$ there is a line $L_{1}^{Y} \in Y$ such that ${\overline{\left[L_{1}\right]}}_{\text {prim }}(Y)=\left[L_{1}^{Y}\right]_{\text {prim }}$. The intersection number of $L_{1}^{Y}$ and $L_{2}^{Y}$ in $Y$ is 1: hence, there is a point $p_{Y} \in L_{1}^{Y} \cap L_{2}^{Y}$. It follows that there is a plane $H_{Y}$ in $\mathbb{P}^{3}$ containing $L_{1}^{Y} \cup L_{2}^{Y}$. Hence, in particular, there is a hyperplane $H_{Y}$ in $\mathbb{P}^{3}$ on which $\gamma_{Y}$ is supported.

In [10] (p. 212, observation 4.a.4) (see also [17], p. 408, proposition 17.19) it is shown that if there exists a holomorphic form $\omega$ on $Y$ such that $\gamma$ is supported on the zero locus of $\omega$ then $\operatorname{codim}\left(T_{N L(\gamma)}(Y)\right)<\left(\begin{array}{c}d-1 \\ 3\end{array}\right)$. Since $K_{Y}=\mathcal{O}_{Y}(1)$, there exists such a holomorphic form, and

$$
\operatorname{codim}\left(T_{N L(\gamma)}(Y)\right)<4
$$

at every point of $N L(\gamma)$. The space $N L(\gamma)$ is therefore non-reduced. This completes the proof of Proposition 1.

We will now prove Theorem 8 , which says that this is the only possible type of non-reduced Noether-Lefschetz locus in $U_{5}$. 
We assume that $X$ is a sufficiently general smooth point of $N L(\gamma)_{\text {red. }}$ Recall that $P$ is a degree 6 polynomial such that $\operatorname{res}_{X}(P)=\gamma$. Since codim $T_{N L(\gamma)}(X)<4$, it follows from the definition of $T_{N L(\gamma)}(X)=\operatorname{Ker}(\cdot P)$ that the map

$$
P: S^{5} \rightarrow R_{F}^{11}
$$

is not surjective. By Macaulay duality there is an $X_{0} \in S^{1}$ such that

$$
X_{0} P H=0 \text { for all } H \in R_{F}^{5},
$$

whence we deduce that $X_{0} P=0$ in $R_{F}$. We define $H$ to be the plane $X_{0}=0$. There exist cubics, $P_{i} \in S^{3}$, such that

$$
X_{0} P=\sum_{i=0}^{3} P_{i} \frac{\partial F}{\partial X_{i}} .
$$

We now use the fundamental quadratic form to obtain relations on the $P_{i}$ and $\frac{\partial F}{\partial X_{i}}$ which will imply that $X \cap H$ is reducible.

4.1. Relationships between $P_{i}$ and $\frac{\partial F}{\partial X_{i}}$. We will now use the fundamental quadratic form to derive some special relationships between the $P_{i} \mathrm{~s}$ and the $\frac{\partial F}{\partial X_{i}} \mathrm{~s}$ (proposition 2). In the following sections, we will use these relationships to prove that $X \cap H$ is reducible.

Proposition 2. We have

$$
\begin{gathered}
\left.\sum_{i=1}^{3} P_{i} \frac{\partial F}{\partial X_{i}}\right|_{H}=0 \\
\left.\sum_{i=1}^{3} \frac{\partial P_{i}}{\partial X_{i}}\right|_{H}=0 .
\end{gathered}
$$

Equation 4 implies immediately that $X \cap H$ is singular. We will prove that in fact the space of triples $P_{1}, P_{2}, P_{3}$ satisfying (4) and (5) has dimension at most $(j-1)$, where $j$ is the number of components of $X \cap H$.

Proof of Proposition 2. We will begin by proving the following lemma.

Lemma 4. There is a non-zero $L$ contained in $S^{1}$ such that in $R_{F}^{4}$

$$
L\left(X_{0} \sum_{i=0}^{3} \frac{\partial P_{i}}{\partial X_{i}}-P_{0}\right)=0 .
$$

Proof of Lemma 4. We know that $\operatorname{codim} T_{N L(\gamma)}(X) \geq 2$ by the result of Voisin and Green, and codim $T_{N L(\gamma)_{\text {red }}}(X)=4$, since $X$ is a smooth point of $N L(\gamma)_{\text {red. }}$. We treat first the case where the codimension of $T_{N L(\gamma)_{\text {red }}}(X)$ in $T_{N L(\gamma)}(X)$ is 1 . We have

$$
\left(X_{0} H\right) P=\sum_{i=0}^{3} P_{i} H \frac{\partial F}{\partial X_{i}}
$$


and similarly

$$
\left(X_{0} G\right) P=\sum_{i=0}^{3} P_{i} G \frac{\partial F}{\partial X_{i}} .
$$

Now, suppose that $G \in S^{4}$ is such that $X_{0} G \in T_{N L(\gamma)_{\text {red }}}(X)$. Then for any $H \in S^{4}$, we have, by remark 1 , that

$$
q_{\bar{\gamma}^{0,2}, X}\left(X_{0} H, X_{0} G\right)=0 .
$$

Hence, the following equations hold in $R_{F}$

$$
X_{0} G \sum_{i=0}^{3} \frac{\partial\left(P_{i} H\right)}{\partial X_{i}}-\sum_{i=0}^{3} P_{i} G \frac{\partial\left(X_{0} H\right)}{\partial X_{i}} \in \operatorname{Im}(\cdot P) .
$$

Rearranging, we get that

$$
G H\left(X_{0} \sum_{i=0}^{3} \frac{\partial P_{i}}{\partial X_{i}}-P_{0}\right) \in \operatorname{Im}(\cdot P) .
$$

Multiplying by $X_{0}$, we get that

$$
X_{0} G H\left(X_{0} \sum_{i=0}^{3} \frac{\partial P_{i}}{\partial X_{i}}-P_{0}\right)=0
$$

and finally, by Macaulay duality, we have

$$
X_{0} G\left(X_{0} \sum_{i=0}^{3} \frac{\partial P_{i}}{\partial X_{i}}-P_{0}\right)=0 .
$$

This last equation holds for any $G$ in the space $E$ defined by

$$
E=\left\{G \in S^{4} \text { such that } X_{0} G \in T_{N L(\gamma)_{\text {red }}}(X)\right\} .
$$

We have that $\operatorname{codim}(E) \leq 1$ (since we have supposed that the codimension of $T_{N L(\gamma)_{\text {red }}}(X)$ in $T_{N L(\gamma)}(X)$ is 1$)$. Straightforward algebraic manipulations show that the ideal generated in $R_{F}$ by $E$ contains $R_{F}^{5}$. Hence for any $J \in R_{F}^{5}$ we have

$$
J X_{0}\left(X_{0} \sum_{i=0}^{3} \frac{\partial P_{i}}{\partial X_{i}}-P_{0}\right)=0
$$

and hence by Macaulay duality

$$
X_{0}\left(X_{0} \sum_{i=0}^{3} \frac{\partial P_{i}}{\partial X_{i}}-P_{0}\right)=0 .
$$

Hence Lemma 4 is proved in this case.

We now treat the case where the codimension of $T_{N L(\gamma)_{\text {red }}}(X)$ in $T_{N L(\gamma)}(X)$ is 
2. In this case, there are two distinct elements of $S^{1}, X_{0}$ and $X_{1}$, such that $X_{0} P=0$ and $X_{1} P=0$. Once again, we define $E$ by

$$
E=\left\{G \in S^{4} \text { such that } X_{0} G \in T_{N L(\gamma)_{\text {red }}}(X)\right\},
$$

and we then obtain that

$$
X_{0} G\left(X_{0} \sum_{i=0}^{3} \frac{\partial P_{i}}{\partial X_{i}}-P_{0}\right)=0
$$

and similarly

$$
X_{1} G\left(X_{0} \sum_{i=0}^{3} \frac{\partial P_{i}}{\partial X_{i}}-P_{0}\right)=0 .
$$

The codimension of $E$ is at most 2. There are 2 maps,

$$
\phi_{0} \text { and } \phi_{1}: S^{4} / E \rightarrow \operatorname{Ker}(\cdot E) \subset R_{F}^{8}
$$

given by multiplication by $X_{0}\left(X_{0} \sum_{i=0}^{3} \frac{\partial P_{i}}{\partial X_{i}}-P_{0}\right)$ and $X_{1}\left(X_{0} \sum_{i=0}^{3} \frac{\partial P_{i}}{\partial X_{i}}-P_{0}\right)$ respectively. Here by $\operatorname{Ker}(\cdot E)$, we mean the set of all elements in $R_{F}^{8}$ which give 0 on multiplying with any element of $E$. If $\phi_{0}$ is not an isomorphism then (7) holds for all $G \in \phi_{0}^{-1}(0)$, which is a hyperplane, and the lemma follows as in the previous case.

Only the case where $\phi_{0}$ is invertible remains. But in this case $\phi_{0}^{-1} \circ \phi_{1}$ has an eigenvalue, $\lambda$. The multiplication map

$$
\cdot\left(X_{0}-\lambda X_{1}\right)\left(X_{0} \sum_{i=0}^{3} \frac{\partial P_{i}}{\partial X_{i}}-P_{0}\right): R_{F}^{4} \rightarrow R_{F}^{8}
$$

has a kernel of codimension at most 1 , from which we conclude as before that $\left(X_{0}-\right.$ $\left.\lambda X_{1}\right)\left(X_{0} \sum_{i=0}^{3} \frac{\partial P_{i}}{\partial X_{i}}-P_{0}\right)=0$. This concludes the proof of Lemma 4.

We will now attempt to prove that this implies that $X_{0} \sum_{i=0}^{3} \frac{\partial P_{i}}{\partial X_{i}}-P_{0}=0$. We start with the following technical lemma.

Lemma 5. If $W^{\prime}$ is defined to be the space $S^{3} \times S^{1} \times\left\{\mathbb{C}^{4} / 0\right\} \times S^{5}$, then the map $\phi: W^{\prime} \rightarrow S^{4}$ given by $\phi\left(P, L, \alpha_{0}, \alpha_{1}, \alpha_{2}, \alpha_{3}, F\right)=P L-\sum_{i=0}^{3} \alpha_{i} \frac{\partial F}{\partial X_{i}}$ is submersive.

Proof of Lemma 5. Let $\left(Y_{0}, \ldots, Y_{3}\right)$ be co-ordinates on $\mathbb{P}^{3}$, such that $\sum_{i=0}^{3} \alpha_{i} \frac{\partial F}{\partial X_{i}}=\frac{\partial F}{\partial Y_{0}}$. Then

$$
\frac{\partial \phi}{\partial F}(G)=\frac{\partial G}{\partial Y_{0}}
$$

Hence $d \phi: T_{W^{\prime}} \rightarrow T_{S^{4}}$ is surjective. This completes the proof of Lemma 5.

From this lemma we will deduce the following:

Lemma 6. If $U^{\prime} \subset U_{5}$ is defined by

$\left\{F\right.$ such that $\exists L_{1} \in R_{F}^{1}, L_{2} \in R_{F}^{3}$ such that $L_{1} \neq 0, L_{2} \neq 0$ and $L_{1} L_{2}=0$ in $\left.R_{F}^{4},\right\}$, 
then $\operatorname{codim} U^{\prime} \geq 6$.

Proof of Lemma 6. We now define $W$ to be the subset of $W^{\prime}$ consisting of all septuples $\left(P, L, \alpha_{0}, \alpha_{1}, \alpha_{2}, \alpha_{3}, F\right)$ such that

$$
P L=\sum_{i=0}^{3} \alpha_{i} \frac{\partial F}{\partial X_{i}} .
$$

It follows that the codimension of $W$ in $W^{\prime}$ is $\operatorname{dim}\left(S^{4}\right)=35$, whence we see that

$$
\operatorname{dim}(W)=\operatorname{dim} S^{5}+4+4+20-35=\operatorname{dim}\left(S^{5}\right)-7 .
$$

It follows that the codimension of the image of $W$ under projection to $U_{5}$ is $\geq 6$. This completes the proof of Lemma 6 .

And finally, this gives us the following.

Lemma 7. In $R_{F}$ we have

$$
X_{0} \sum_{i=0}^{3} \frac{\partial P_{i}}{\partial X_{i}}-P_{0}=0 .
$$

Proof of Lemma \%. Indeed, it follows immediately from Lemma 6, and the fact that

$$
\operatorname{codim}\left(N L(\gamma)_{\text {red }}\right)=4,
$$

that for a generic point of $N L(\gamma)(6)$ implies that

$$
X_{0} \sum_{i=0}^{3} \frac{\partial P_{i}}{\partial X_{i}}-P_{0}=0 .
$$

So Lemma 7 follows from Lemma 6 . This completes the proof of Lemma 7.

Equation (4) of Proposition 2 now follows from the two equations

$$
P_{0}=X_{0} \sum_{i=0}^{3} \frac{\partial P_{i}}{\partial X_{i}}
$$

and

$$
\sum_{i=0}^{3} P_{i} \frac{\partial F}{\partial X_{i}}=X_{0} P
$$

We turn now to the equation (5), which follows when we differentiate (9) with respect to $X_{0}$ to obtain

$$
\frac{\partial P_{0}}{\partial X_{0}}=\sum_{i=0}^{3} \frac{\partial P_{i}}{\partial X_{i}}+X_{0} \frac{\partial\left(\sum_{i=0}^{3} \frac{\partial P_{i}}{\partial X_{i}}\right)}{\partial X_{0}}
$$


Re-arranging, we get that

$$
-X_{0} \frac{\partial \sum_{i=0}^{3} \frac{\partial P_{i}}{\partial X_{i}}}{\partial X_{0}}=\sum_{i=1}^{3} \frac{\partial P_{i}}{\partial X_{i}} .
$$

This completes the proof of Proposition 2.

Now, let us consider the quintic plane curve, $D=X \cap H$. In the next section, we will denote by $\tilde{F}$ the restriction of $F$ to $H$. We define $D_{1}, \ldots, D_{j}$ to be the components of $D$ and $d_{i}$ to be the degree of $D_{i}$.

4.2. The cohomology class $\gamma$ is a linear combination of $\left[D_{1}\right], \ldots,\left[D_{j}\right]$. We will show that the dimensions of the following two spaces are the same :

1. Triples $P_{i}$ satisfying the equations of Proposition 2,

2. Primitive cohomology classes supported on $D$.

From this, it will not be too hard to show that $\gamma$ is supported on $D$. We now prove the following proposition.

Proposition 3. The cohomology class $\gamma$ is a linear combination of $\left[D_{1}\right], \ldots,\left[D_{j}\right]$.

Proof of Proposition 3. It will be enough to show that

$$
\operatorname{dim}\left(\left\langle\gamma,\left[D_{1}\right]_{\text {prim }}, \ldots,\left[D_{j-1}\right]_{\text {prim }}\right\rangle\right) \leq j-1 .
$$

We denote this space by $V^{\prime}$. We denote by $V$ the space of all triplets of cubics $\left(P_{1}, P_{2}, P_{3}\right)$ in variables $X_{1}, X_{2}, X_{3}$ such that

$$
\sum_{i=1}^{3} P_{i} \frac{\partial \tilde{F}}{\partial X_{i}}=0
$$

and

$$
\sum_{i=1}^{3} \frac{\partial P_{i}}{\partial X_{i}}=0
$$

Of course, these are simply the equations of Proposition 2. We will first show that the dimension of $V$ is less than or equal to $(j-1)$ and then construct an injective linear map $V^{\prime} \rightarrow V$, from which (10) will follow.

Lemma 8. The dimension of $V$ is $\leq j-1$.

Proof of Lemma 8. For this, we will need to interpret the equations (11) and (12) geometrically. We consider the maps

$$
f: V \rightarrow H^{0}\left(T_{\mathbb{P}^{2}}(2)\right)
$$

and

$$
g: H^{0}\left(T_{\mathbb{P}^{2}}(2)\right) \rightarrow H^{0}\left(\Omega_{\mathbb{P}^{2}}(D)\right)
$$

which are given by

$$
f\left(P_{1}, P_{2}, P_{3}\right)=\sum_{i=1}^{3} P_{i} \frac{\partial}{\partial X_{i}}
$$


and

$$
g(\alpha)=\frac{\operatorname{int}(\alpha) \Omega}{\tilde{F}} .
$$

The map int is as given on page 10 . In this case, $\Omega$ is the canonical section of $K_{\mathbb{P}^{2}}(3)$. The map $g$ is an isomorphism. We will show the following lemma.

Lemma 9. The map $f$ is injective.

Proof of Lemma 9. Suppose that the triple $\left(P_{1}, P_{2}, P_{3}\right)$ were such that $f\left(P_{1}, P_{2}, P_{3}\right)=0$. There would then be $P^{\prime}$ such that

$$
\left(P_{1}, P_{2}, P_{3}\right)=\left(X_{1} P^{\prime}, X_{2} P^{\prime}, X_{3} P^{\prime}\right) .
$$

However we would then have

$$
\sum_{i=1}^{3} P_{i} \frac{\partial \tilde{F}}{\partial X_{i}}=P^{\prime} \tilde{F}
$$

and hence (11) implies that $P^{\prime}=0$. This completes the proof of Lemma 9 .

We now consider the image of $g \circ f$ in $H^{0}\left(\Omega_{\mathbb{P}^{2}}(D)\right)$. We will use the following lemma.

Lemma 10. If $\left(P_{1}, P_{2}, P_{3}\right) \in V$ then $g \circ f\left(P_{1}, P_{2}, P_{3}\right) \in H^{0}\left(\Omega_{\mathbb{P}^{2}}^{1, c}(\log D)\right)$.

Here, $\Omega_{\mathbb{P}^{2}}^{1, c}(\log D)$ denotes the sheaf of closed differential forms with logarithmic singularities along $D$. We note that, since differential forms with logarithmic singularities can be characterised as being those differential forms with simple poles along $D$ whose differential also has logarithmic poles along $D$, it is automatic that any $d$-closed member of $H^{0}\left(\Omega_{\mathbb{P}^{2}}^{1, c}(D)\right.$ has in fact a logarithmic singularity along $D$.

Proof of Lemma 10. It is enough to show that $d\left(g \circ f\left(P_{1}, P_{2}, P_{3}\right)\right)=0$. But

$$
d\left(\frac{\sum_{i=1}^{3}\left(P_{i} \operatorname{int}\left(\frac{\partial}{\partial X_{i}}\right)(\Omega)\right)}{\tilde{F}}\right)=\sum_{i=1}^{3} \frac{\left(-P_{i} \frac{\partial \tilde{F}}{\partial X_{i}}+\tilde{F} \frac{\partial P_{i}}{\partial X_{i}}\right) \Omega}{\tilde{F}^{2}} .
$$

By (11) and (12), the right hand side is 0. This completes the proof of Lemma 10.

We now complete the proof of Lemma 8. By the above, $V$ injects into $H^{0}\left(\Omega_{\mathbb{P}^{2}}^{1, c}(\log D)\right)$. Note that $D$, being the intersection of a smooth surface and a plane, is reduced.

We define $U$ to be $\mathbb{P}^{2}-D_{\text {sing. }}$. By the above comment, $U$ is $\mathbb{P}^{2}$ minus a codimension 2 subset. There is an exact sequence on $U$,

$$
0 \rightarrow \Omega_{U}^{1, c} \rightarrow \Omega_{U}^{1, c}(\log D) \stackrel{\text { res }}{\rightarrow} \mathbb{C}_{D-D_{\text {sing }}} \rightarrow 0,
$$

from which we get an associated long exact sequence,

$$
H^{0}\left(\Omega_{U}^{1, c}\right) \rightarrow H^{0}\left(\Omega_{U}^{1, c}(\log D)\right) \stackrel{p}{\rightarrow} H^{0}\left(D / D_{\text {sing }}, \mathbb{C}\right) \stackrel{\delta}{\rightarrow} H^{1}\left(\Omega_{U}^{1, c}\right) .
$$


However, since $\Omega_{\mathbb{P}^{2}}^{1}$ is free and $\mathbb{P}^{2}-U$ is of codimension 2, it follows by Levi's extension theorem that

$$
H^{0}\left(\Omega_{U}^{1}\right) \simeq H^{0}\left(\Omega_{\mathbb{P}^{2}}^{1}\right)=0
$$

Hence,

$$
H^{0}\left(\Omega_{U}^{1, c}(\log D)\right) \simeq \operatorname{Ker} \delta .
$$

Since $\operatorname{dim}\left(H^{0}\left(D / D_{\text {sing }}, \mathbb{C}\right)\right)=j$, it will be enough to show that $\operatorname{Im}(p) \neq H^{0}(D-$ $\left.D_{\text {sing }}, \mathbb{C}\right)$. But if $u \in H^{0}\left(\Omega_{U}^{1, c}(\log D)\right)$ then we have that

$$
p(u)\left(D_{i}\right)=\operatorname{res}_{D_{i}}(u)
$$

where $\operatorname{res}_{D_{i}}(u)$ is the residue of the form $u$ along $D_{i}$. But we know that $\sum_{i=1}^{j} d_{i} \operatorname{res}_{D_{i}} u=0$ and from this it follows that

$$
\operatorname{dim}\left(H^{0}\left(\Omega_{\mathbb{P}^{2}}^{1, c}(\log D)\right)\right) \leq j-1 .
$$

This completes the proof of Lemma 8 .

We now prove the following lemma.

Lemma 11. The space $V^{\prime}$ has dimension $\leq j-1$.

Proof of Lemma 11. We will construct a map $L: V^{\prime} \rightarrow V$ which we will then show to be injective. We choose a basis $\left(e_{1} \ldots, e_{m}\right)$ for $V^{\prime}$, such that

1. $e_{1}=\gamma$

2. $e_{2}, \ldots, e_{m} \in\left\langle\left[D_{1}\right]_{\text {prim }}, \ldots,\left[D_{j-1}\right]_{\text {prim }}\right\rangle$.

We will show that the argument presented in the proof of Proposition 2 is also valid for polynomials representing classes in the space

$$
\left\langle\left[D_{1}\right]_{\text {prim }}, \ldots,\left[D_{j-1}\right]_{\text {prim }}\right\rangle .
$$

For each $e_{l}$, we choose $Q^{l}$, a degree 6 polynomial such that $\operatorname{res}_{X}\left(Q^{l}\right)=e_{l}$. By the choice of basis, we have the following.

Lemma 12. For all $l, X_{0} Q^{l}=0$ in $R_{F}^{7}$.

Proof of Lemma 12. This is true for $e_{1}=\gamma$ by definition. For $l \geq 2$, it follows from

$$
e_{l} \in\left\langle\left[D_{1}\right]_{\text {prim }}, \ldots,\left[D_{j-1}\right]_{\text {prim }}\right\rangle
$$

that

$$
X_{0} \cdot S^{4} \subset T_{N L\left(e_{l}\right)}(X) .
$$

This, by Macaulay duality and the results of Carlson and Griffiths, is equivalent to $X_{0} Q^{l}=0$ in $R_{F}$. This completes the proof of Lemma 12 .

We now choose polynomials $Q_{0}^{l}, Q_{1}^{l}, Q_{2}^{l}, Q_{3}^{l}$ (in four variables) such that

$$
X_{0} Q^{l}=\sum_{i=0}^{3} Q_{i}^{l} \frac{\partial F}{\partial X_{i}} .
$$


We then have the following lemma.

LEMma 13. The equation (6) is valid for $\left(Q_{0}^{l}, \ldots, Q_{3}^{l}\right)$. The equations (11) and (12) are valid for the triple $\left(\left.Q_{1}^{l}\right|_{H}, \ldots,\left.Q_{3}^{l}\right|_{H}\right)$.

Proof of Lemma 13. For $l=1$, this is the statement of Proposition 2. For $l \geq 2$, Lemma 12 implies that for all degree 4 polynomials $G_{1}$ and $G_{2}$,

$$
X_{0} G_{1}, X_{0} G_{2} \in T_{N L\left(e_{l}\right)_{\mathrm{red}}}(X) .
$$

Hence we see that for all $G_{1}$ and $G_{2}$ in $S^{4}$,

$$
q_{\bar{e}_{l}, 2, X}\left(X_{0} G_{1}, X_{0} G_{2}\right)=0 .
$$

Alternatively, as in the proof of Proposition 2

$$
G_{1} G_{2}\left(X_{0} \sum_{i=0}^{3} \frac{\partial Q_{i}^{l}}{\partial X_{i}}-Q_{0}^{l}\right) \in \operatorname{Im}(\cdot P)
$$

and multiplying by $X_{0}$ we see that

$$
X_{0} G_{1} G_{2}\left(X_{0} \sum_{i=0}^{3} \frac{\partial Q_{i}^{l}}{\partial X_{i}}-Q_{0}^{l}\right)=0
$$

in $R_{F}$ This time, this relationship is valid for any choice of $G_{1}$ and $G_{2}$, so it follows immediately by Macaulay duality that

$$
X_{0}\left(X_{0} \sum_{i=0}^{3} \frac{\partial Q_{i}^{l}}{\partial X_{i}}-Q_{0}^{l}\right)=0
$$

in $R_{F}$. This is precisely equation (6). By Lemma 7 , it follows that since $X$ has been chosen general in $N L(\gamma)$

$$
\left(X_{0} \sum_{i=0}^{3} \frac{\partial Q_{i}^{l}}{\partial X_{i}}-Q_{0}^{l}\right)=0
$$

in $R_{F}$. Indeed, since $\operatorname{deg}\left(Q_{0}^{l}\right)=3$, it follows that $\left(X_{0} \sum_{i=0}^{3} \frac{\partial Q_{i}^{l}}{\partial X_{i}}-Q_{0}^{l}\right)=0$. The two equations (11) and (12) now follow as in the proof of Proposition 2. This completes the proof of Lemma 13.

We set $L\left(e_{l}\right)=\left(\left.Q_{1}^{l}\right|_{H},\left.Q_{2}^{l}\right|_{H},\left.Q_{3}^{l}\right|_{H}\right)$ and extend by linearity. We will now prove the following lemma.

Lemma 14. $L$ is injective.

Proof of Lemma 14. Let $v$ be any element of $V^{\prime}$. By linearity, there are cubic polynomials $Q_{0}^{v}, Q_{1}^{v}, Q_{2}^{v}, Q_{3}^{v}$ in variables $X_{0}, \ldots, X_{3}$ such that

1. $L(v)=\left(\left.Q_{1}^{v}\right|_{H},\left.Q_{2}^{v}\right|_{H},\left.Q_{3}^{v}\right|_{H}\right)$,

2. The equation (6) is valid for $Q_{0}^{v}, \ldots, Q_{3}^{v}$,

3. There exists a $Q^{v}$ such that $\sum_{i=0}^{3} Q_{i}^{v} \frac{\partial F}{\partial X_{i}}=X_{0} Q^{v}$,

4. $Q^{v}$ represents the cohomology class $v$. 
Lemma 14 now follows from the following lemma.

Lemma 15. Suppose that $\gamma=\operatorname{res}_{X}(P)$, and there exist $\left(P_{0}, \ldots, P_{3}\right)$ such that

$$
X_{0} P=\sum_{i=0}^{3} P_{i} \frac{\partial F}{\partial X_{i}} .
$$

Suppose further that (6) is valid and that

$$
\left.P_{1}\right|_{H}=\left.P_{2}\right|_{H}=\left.P_{3}\right|_{H}=0, i \geq 1 .
$$

Then $\gamma^{1,1}=0$.

Proof of Lemma 15. We have

$$
X_{0} P=\sum_{i=0}^{3} P_{i} \frac{\partial F}{\partial X_{i}} .
$$

By hypothesis, $X_{0}$ divides $P_{i}$ for $i \geq 1$. It follows from (6) that $X_{0}$ divides $P_{0}$. Therefore, (14) implies that

$$
P \in\left\langle\frac{\partial F}{\partial X_{i}}\right\rangle
$$

from which it follows that

$$
\operatorname{res}_{X} P \in F^{2}\left(H^{2}(X, \mathbb{C})\right) .
$$

Alternatively, we have that

$$
\gamma^{1,1}=0 .
$$

This completes the proof of Lemma 15.

Since all elements of $V^{\prime}$ are Hodge $(1,1)$ classes, the injectivity of $L$ follows immediately. This completes the proof of Lemma 19.

This completes the proof of Lemma 11.

This completes the proof of Proposition 3.

4.3. The curve $D$ is generically the union of two lines and a cubic. To complete the theorem, it will be enough to show that $D$ is necesarily the union of two lines and a (possibly reducible) cubic. This will follow from a simple dimension count.

Lemma 16. The curve $X \cap H$ must have at least 3 components.

Proof of Lemma 16. We know that $\gamma$ is a linear combination of classes of curves contained on $X \cap H$. If $X \cap H$ contains only two reducible components, then $\gamma$ is either the linear combination of

1. a line and a hyperplane section or

2. a conic and a hyperplane section. 
This is not possible, since all such cohomology classes have reduced associated Noether-Lefschetz loci. This completes the proof of Lemma 16.

There are now two possibilities:

1. $\gamma$ is a linear combination of the cohomology classes of two lines and a hyperplane section,

2. $X$ belongs to $S$, the space of all quintic hypersurfaces possessing a hyperplane section which is the union of two conics and a line.

The codimension of $S$ is 5 and the codimension of $N L(\gamma)$ is at most 4, so the general element of $N L(\gamma)$ cannot be contained in $S$.

It remains only to exclude the cases $\gamma=\alpha\left(\left[L_{1}+L_{2}\right]_{\text {prim }}\right)$ or $\gamma=\alpha\left(\left[L_{1}\right]_{\text {prim }}\right)$. In the first case, $\gamma$ is (a multiple of) the primitive part of the cohomology class of a conic, and in the second case $\gamma$ is (a multiple of) the primitive part of the cohomology class of a line. In either case, $\gamma$ has a reduced Noether-Lefschetz locus.

This concludes the proof of Theorem 2 .

5. A weaker form of the Green-Ciliberto conjecture holds (proof of Theorem 3). Let us begin by summarising the motivation for the Green-Ciliberto conjecture. We recall that the tangent space $T_{N L(\gamma)}(X)$ is simply the kernel of the map

$$
P: S^{d} / F \rightarrow R_{F}^{3 d-4}
$$

which is multiplication by $P$. If $N L(\gamma)$ is exceptional, then the multiplication map $\cdot P: R_{F}^{d} \rightarrow R_{F}^{3 d-4}$ is not onto. Since the multiplication map

$$
R_{F}^{d-4} \otimes R_{F}^{3 d-4} \rightarrow R_{F}^{4 d-8}
$$

is a perfect pairing this is equivalent to saying that there exists $Q \in S^{d-4}$ such that $Q P=0$ in $R_{F}$. This is equivalent to saying that

$$
Q \cdot S^{4} \subset T_{N L(\gamma)}(X) .
$$

There is one case in which it is clear this will be the case - namely when $\gamma$ is supported on $Z \cap X$, where $Z$ is the surface defined by $Q$. (In this case, we will say that $\gamma$ is supported on $Q$ ). The Green-Ciliberto conjecture says that this should be the only possibility. The main theorem of this section is as follows.

TheOrEm 9. Suppose that $e \leq \frac{d-1}{2}$ and $j \leq\left(\begin{array}{c}e+3 \\ 3\end{array}\right)$. There exists an integer, $\phi_{e, j}(d)$ such that if $N L(\gamma)$ is reduced and codim $(N L(\gamma)) \leq \phi_{e, j}(d)$ then the dimension of the space $\left\{Q \in S^{e}\right.$ such that $\gamma$ is supported on $\left.Q\right\}$ is $\geq j$.

Further, $\phi_{\frac{d-1}{2}, 1}(d)=O\left(d^{3}\right)$.

On setting $j=1$ in this statement, we obtain the result given in the introduction.

5.1. Integrating along special sub-bundles of $T_{N L(\gamma)}$. One way in which one might thnk of trying to prove that the class $\gamma$ is supported on $Q$ would be to try to show that $F+G Q$ is contained in $N L(\gamma)$. From this it would follow by a degeneration argument - due to Griffiths and Harris for smooth $Q$, and Voisin for general $Q$ - that $\gamma$ is supported on $Q$. 
This is equivalent to showing that under small perturbation of $F$ in the direction $t G Q$ the tangent vector $G Q$ does not leave the tangent space $T_{N L(\gamma)}$. Unfortunately, this is false. However, in what follows, we show that under the condition that $Q \cdot S^{d-e} \subset T_{N L(\gamma)}(X)$, with $e \leq \frac{d-1}{2}$, we have that $F+G Q^{2}$ is contained in $N L(\gamma)$ for any $G$.

The theorem will follow immediately from the following two propositions.

Proposition 4. Suppose that $N L(\gamma)$ is reduced and for all $Y$ in some neighbourhood of $X$, a general element of $N L(\gamma)$, the space

$$
V=\left\{Q \in S^{e} \mid Q \cdot S^{d-e} \subset T_{N L(\gamma)}(Y)\right\}
$$

is of dimension $j>0$. Suppose further that $e \leq \frac{d-1}{2}$. Then, for all $Q \in V$ and $G \in S^{d-2 e}$ such that $F+G Q^{2} \in O$ we have $F+G Q^{2} \in N L(\gamma)$.

Proposition 5. Let $X$ be an element of $N L(\gamma)$. We can construct $\phi_{e, j}(d)$ as above such that if

$$
\operatorname{codim}(N L(\gamma)) \leq \phi_{e, j}(d)
$$

then $\operatorname{dim}\left\{Q \in S^{e} \mid Q \cdot S^{d-e} \subset T_{N L(\gamma)}(X)\right\} \geq j$.

Given these two propositions, it follows by the argument given in section 2 of [15], (pp 56-59), that $\gamma$ is supported on $Q^{2}=0$ - and hence on $Q=0$.

Proof of Proposition 4. We assume, since the question was dealt with for $d=6,7$ in [15], that $d \geq 8$. We construct a space $W$ as follows:

$$
W=\left\{(Y, A) \in N L(\gamma) \times S^{e} \mid A \cdot S^{d-e} \subset T_{N L(\gamma)}(Y)\right\} .
$$

If $X$ is a sufficiently general smooth point of $N L(\gamma)$, then the space

$$
V_{Y}=\left\{A \in S^{e} \mid A \cdot S^{d-e} \subset T_{N L(\gamma)}(Y)\right\}
$$

is of constant dimension near $X$. The space $W$ will be a smooth over some neighbourhood of $X$. We will prove the following lemma.

Lemma 17. At any point $(Y, A)$ of $W$ we have $\left(G A^{2}, 0\right) \in T_{W}(Y, A)$ for all $G$.

Proof of Lemma 17. We know that there exists some $B$ such that $\left(G A^{2}, B\right) \in$ $T_{W}(Y, A)$, since the map $W \rightarrow N L(\gamma)$ locally induces a surjection on the tangent spaces. Denote this tangent vector by $\chi$. Let us derive the equation

$$
A P=\sum_{i} L_{i} \frac{\partial F}{\partial X_{i}}
$$

in the direction $\chi$. By Lemma 3, we can choose to have that

$$
\chi(P)=\sum_{i} \frac{\partial\left(L_{i} G A\right)}{\partial X_{i}} .
$$

By definition of $\chi$ we have $\chi(A)=B$ and $\chi(F)=\left(G A^{2}\right)$. Hence we have

$$
A \sum_{i}\left(\frac{\partial\left(L_{i} G A\right)}{\partial X_{i}}\right)+B P=\sum_{i}\left(L_{i} \frac{\partial G A^{2}}{\partial X_{i}}+\chi\left(L_{i}\right) \frac{\partial F}{\partial X_{i}}\right) .
$$


Rearranging, we get that in $R_{F}$

$$
G A \sum_{i}\left(A \frac{\partial L_{i}}{\partial X_{i}}-L_{i} \frac{\partial A}{\partial X_{i}}\right)=-B P .
$$

We will now prove the following result.

Lemma 18. We have

$$
A \sum_{i}\left(A \frac{\partial L_{i}}{\partial X_{i}}-L_{i} \frac{\partial A}{\partial X_{i}}\right)=0 \text { in } R_{F} .
$$

Proof of Lemma 18. It is in the proof of this key lemma that we will use the fundamental quadratic form. Note that for all $H_{1}, H_{2} \in S^{d-e}$,

$$
A H_{1} \text { and } A H_{2} \in T_{N L(\gamma)}(X) \text {, }
$$

and further,

$$
q_{\bar{\gamma}^{0,2}, X}\left(A H_{1}, A H_{2}\right)=0 .
$$

Hence, for all $H_{1}, H_{2}$ the following equality holds in $R_{F}$

$$
\sum_{i}\left(A H_{1} \frac{\partial\left(H_{2} L_{i}\right)}{\partial X_{i}}-H_{1} L_{i} \frac{\partial\left(A H_{2}\right)}{\partial X_{i}}\right) \in \operatorname{Im}(\cdot P) .
$$

Rearranging, we get that

$$
H_{1} H_{2} \sum_{i}\left(A \frac{\partial L_{i}}{\partial X_{i}}-L_{i} \frac{\partial A}{\partial X_{i}}\right) \in \operatorname{Im}(\cdot P) .
$$

From this we see that for all $H \in S^{2 d-2 e}$,

$$
H A \sum_{i}\left(A \frac{\partial L_{i}}{\partial X_{i}}-L_{i} \frac{\partial A}{\partial X_{i}}\right)=0
$$

in $R_{F}$. We know that

$$
\operatorname{deg} H A \sum_{i}\left(A \frac{\partial L_{i}}{\partial X_{i}}-L_{i} \frac{\partial A}{\partial X_{i}}\right)=3 d-4+e \leq 4 d-8 .
$$

In the last inequality we have used the fact that $d \geq 8$. It follows that

$$
A \sum_{i}\left(A \frac{\partial L_{i}}{\partial X_{i}}-L_{i} \frac{\partial A}{\partial X_{i}}\right)=0
$$

in $R_{F}$. This completes the proof of Lemma 18 .

Returning to the proof of Lemma 17 , we see that $B P=0$. Hence

$$
(0, B) \in T_{W}(Y, A)
$$


and therefore

$$
\left(G A^{2}, 0\right) \in T_{W}(Y, A) \text { for all } G \in S^{d-2 e} .
$$

This completes the proof of Lemma 17.

We now complete the proof of Proposition 4. We have just shown there is a field of tangent vectors on $W$ which we denote by $\tau_{G}$ given by

$$
\tau_{G}(Y, A)=\left(G A^{2}, 0\right) .
$$

We may now integrate along the tangent field $\tau_{G}$, at least locally. (Here, we have used the fact that $(Y, A)$ is a smooth point of $W)$. Hence $F+\epsilon G p^{2}$ is contained in $N L(\gamma)$ for all sufficiently small $\epsilon$. This completes the proof of Proposition 4 .

We must now construct the integer $\phi_{e, j}(d)$ such that if $\operatorname{codim}(N L(\gamma)) \leq \phi_{e, j}(d)$ then the dimension of the space

$$
V=\left\{Q \in S^{e} \text { such that } Q \cdot S^{d-e} \in T_{N L(\gamma)}(X)\right\}
$$

is at least $j$. In what follows, when $W \subset S^{n}$ is a sub-vector space, $\langle V\rangle^{n+m}$ will denote the subspace of $S^{n+m}$ generated by $W$.

Proof of Proposition 5. This theorem is essentially a statement about multiplication in a certain polynomial ring. We will rely on the following theorem, due to Macaulay and Gotzmann which may be found in [5] (pp. 64-65).

Theorem 10 (Macaulay, Gotzmann). Given an integer, $d$, any other integer c may be written in a unique way as

$$
c=\left(\begin{array}{c}
k_{d} \\
d
\end{array}\right)+\left(\begin{array}{c}
k_{d-1} \\
d-1
\end{array}\right)+\ldots\left(\begin{array}{c}
k_{i} \\
i
\end{array}\right)
$$

for some integer $i$. where $k_{d}>k_{d-1} \cdots>k_{i}$. We define $c^{<d>}$ by

$$
c^{<d>}=\left(\begin{array}{c}
k_{d}+1 \\
d+1
\end{array}\right)+\left(\begin{array}{c}
k_{d-1}+1 \\
d
\end{array}\right)+\ldots\left(\begin{array}{c}
k_{i}+1 \\
i+1
\end{array}\right) .
$$

Let $V$ be a subvector space of $S^{d}$ of codimension c. Then, the codimension of $\langle V\rangle^{d+1}$ in $S^{d+1}$ is $\leq c^{<d>}$ and if equality holds then for all $j$ we have

$$
\operatorname{codim}\left(\langle V\rangle^{d+j}\right)=\left(\left(\left(c^{<d>}\right)^{<d+1>}\right) \ldots\right)^{<d+j-1>} .
$$

Here, $\langle V\rangle^{i}$ denotes the degree $i$ part of the ideal generated by $V$ in $\mathbb{C}\left[X_{0}, \ldots X_{3}\right]$. We now define a set of functions, $g_{i}(n)$. The function $g_{i}(n)$ should be thought of as "the maximal codimension of $\langle V\rangle^{d+i}$ in $S^{d+i}$ if $V$ is a subvector space of $S^{d}$ of codimension $n$ containing $\left\langle\frac{\partial F}{\partial X_{0}}, \ldots, \frac{\partial F}{\partial X_{3}}\right\rangle$." We define

- $g_{0}(n)=n$,

- $g_{i+1}(n)=g_{i}(n)^{<d+i>}-1$.

Lemma 19. If $V \subset S^{d}$ has codimension $n$ and $S^{1} \cdot\left\langle\frac{\partial F}{\partial X_{i}}\right\rangle \subset V$, then for any integer $j$ the subspace generated by $V$ in $S^{d+j}$ has codimension $\leq g_{j}(n)$. 
Proof of Lemma 19. This follows from Theorem 10 by induction on noting that the inclusion $S^{1} \cdot\left\langle\frac{\partial F}{\partial X_{i}}\right\rangle \subset V$ implies that $V$ generates $S^{4 d-7}$, and hence it is not possible to have

$$
\operatorname{codim}\left(\langle V\rangle^{d+j+1}\right)=\left(\operatorname{codim}\left(\langle V\rangle^{d+j}\right)\right)^{<d+j\rangle}
$$

for any $j \leq 3 d-8$. This completes the proof of Lemma 19 .

We are now in a position to define the integer $\phi_{e, j}(d)$.

DEFINITION 2. The integer $\phi_{e, j}(d)$ is the smallest integer $n$ having the property that

$$
g_{2 d-4-e}(n) \leq\left(\begin{array}{c}
e+3 \\
3
\end{array}\right)-j
$$

The above work can be combined to prove Theorem 9 with this definition of $\phi_{e, j}$. It will be enough to show that if $\operatorname{codim}(N L(\gamma)) \leq \phi_{e, j}(d)$ then $\operatorname{dim} \operatorname{Ker}(\cdot P) \geq j$. But the ring

$$
S_{F}=R_{F} / \operatorname{Ker}(\cdot P)
$$

is a Gorenstein graded ring of rang $2 d-4$. It follows by duality that

$$
\operatorname{dim}\left(S_{F}\right)^{e}=\operatorname{dim}\left(S_{F}\right)^{2 d-4-e}
$$

and hence that

$$
\operatorname{dim}\left(R_{F} / \operatorname{Ker}(\cdot P)\right)^{e} \leq\left(\begin{array}{c}
e+3 \\
3
\end{array}\right)-j
$$

by the definition of $\phi_{e, j}(d)$. Hence we have

$$
\operatorname{dim}(\operatorname{Ker}(\cdot P))^{e} \geq j .
$$

REMARK 2. When we choose $e=1, j=2$, we recover the result of [13] and [6] albeit with the additional hypothesis that $N L(\gamma)$ should be reduced.

It remains only to prove that $\phi_{\frac{d-1}{2}}(d)$ is indeed a cubic function of $d$.

Proposition 6. There exists $\alpha>0$ such that

$$
\phi_{\frac{d-1}{2}}(d) \geq \alpha d^{3}
$$

for d sufficiently large.

Proof of Proposition 6. Since $\left(\frac{d-1}{2}+3\right)$ is a cubic in $d$, there exists $\beta<1$ such that for $d$ large

$$
\left(\begin{array}{c}
\frac{d-1}{2}+3 \\
3
\end{array}\right)-1 \geq(\beta d+1)\left(\begin{array}{c}
\frac{3 d-1}{2}+2 \\
2
\end{array}\right)
$$


Hence

$$
\left(\begin{array}{c}
\frac{d-1}{2}+3 \\
3
\end{array}\right)-1 \geq \sum_{i=0}^{\lceil\beta d\rceil}\left(\begin{array}{c}
\frac{3 d-1}{2}-i+2 \\
2
\end{array}\right)
$$

and it follows that

$$
g_{\frac{d+1}{2}}\left(\sum_{i=0}^{\lceil\beta d\urcorner}\left(\begin{array}{c}
d-i+2 \\
2
\end{array}\right)\right) \leq\left(\begin{array}{c}
\frac{d-1}{2}+3 \\
3
\end{array}\right)-1 .
$$

Hence we have

$$
\phi_{\frac{d-1}{2}, 1}(d) \geq \sum_{i=0}^{\lceil\beta d\rceil}\left(\begin{array}{c}
d-i+2 \\
2
\end{array}\right) .
$$

But we know that

$$
\sum_{i=0}^{\lceil\beta d\urcorner}\left(\begin{array}{c}
d-i+2 \\
2
\end{array}\right)>\frac{\beta(1-\beta)}{2} d^{3} .
$$

and this completes the proof of Proposition 6.

Theorem 8 follows immediately.

\section{REFERENCES}

[1] J. Carlson, P. Griffiths, Infinitesimal variations of Hodge structure and the global Torelli problem, Géométrie Algébrique, ed. A. Beauville, Sijthoff-Noordhoff, Angers 1980, pp. 5176 .

[2] J. Carlson, M. Green, P. Griffiths, J. Harris, Infinitesimal variations of Hodge structure (1), Compositio Mathematica, 50 (1983), no.2-3, pp. 109-205.

[3] C. Ciliberto, J. Harris, R. Miranda, General components of the Noether-Lefschetz locus and their density in the space of all surfaces, Math. Ann., 282:4 (1988), pp. 667-680.

[4] D. Eisenbud, Commutative Algebra (with a view towards Algebraic Geometry), Springer, Graduate Texts in Mathematics, 150.

[5] G. Gotzmann, Eine Bedingung für die Flachheit und das Hilbert polynom eines Graduierten Ringes, Math. Z., 158:1 (1978), pp. 61-70.

[6] M. Green, Components of maximal dimension in the Noether-Lefschetz locus, J. Diff, Geom., 29:2 (1989), pp. 295-302.

[7] P. Griffiths, Periods of integrals on algebraic manifolds, 2, American Journal of Mathematics, 90 (1968), pp. 805-865.

[8] P. Griffiths, On the periods of certain rational integrals I, II, Ann. of Math., 90:2 (1969), pp. 460-495; ibid., 90:2 (1969), pp. 496-541.

[9] P. Griffiths, J. HARRIS, On the Noether-Lefschetz locus and some remarks on codimensiontwo cycles, Math. Ann, 271:1 (1985), pp. 31-51.

[10] P. Griffiths, J. HARris, Infinitesimal variations of Hodge structure (2), Compositio Mathematica, 50 (1983), no. 2-3, pp. 109-205.

[11] A. Отwinowska, Composantes de petite codimension du lieu de Noether-Lefschez : un argument asymptotique en faveur de la conjecture de Hodge, J. Alg. Geom., 12:2 (2003), pp. 307-320.

[12] A. Otwinowska, Monodromie d'une famille d'hypersurfaces ; application à l'étude du lieu de Noether-Lefschetz, submitted for publication.

[13] C. Voisin, Une précision concernant le théorème de Noether, Math. Ann., 280:4 (1988), pp. 605-611.

[14] C. Voisin, Composantes de petite codimension du lieu de Noether-Lefschetz, Comm. Math. Helve., 64:4 (1989), pp. 515-526. 
[15] C. Voisin, Sur le lieu de Noether-Lefschetz en degrés 6 et 7, Compositio Mathematica, 75:1 (1990), pp. 47-68.

[16] C. Voisin, Contrexemple à une conjecture de J. Harris, C.R. Acad. Sci. Paris Sér. I Math., 313 (1991), pp. 685-687.

[17] C. Voisin, Théorie de Hodge et géométrie algébrique complexe, Cours Spécialisés, 10. Société Mathématique de France, Paris, 2002. 
C. MACLEAN 